\title{
Developing A Risk Stratification Tool For Predicting Opioid-Related Respiratory Depression After Noncardiac Surgery
}

\section{Sounak Roy}

Vanderbilt University Medical Center

Stephen Bruehl

Vanderbilt University Medical Center

\section{Xiaoke Feng}

Vanderbilt University Medical Center

Matthew S. Shotwell

Vanderbilt University Medical Center

Thomas Van De Ven

Duke University

Andrew D. Shaw

Cleveland Clinic

Miklos D. Kertai ( $\triangle$ miklos.kertai@vumc.org )

Vanderbilt University Medical Center

\section{Research Article}

Keywords: naloxone, respiratory depression, post-operative pain management, non-cardiac surgery

Posted Date: February 28th, 2022

DOI: https://doi.org/10.21203/rs.3.rs-1370010/v1

License: (1) (1) This work is licensed under a Creative Commons Attribution 4.0 International License. Read Full License 


\section{Abstract}

Background: Accurately assessing the probability of significant respiratory depression following opioid administration can potentially enhance perioperative risk assessment and pain management. We developed and validated a risk prediction tool to estimate the probability of significant respiratory depression (indexed by naloxone administration) in patients undergoing noncardiac surgery.

Methods: We studied $n=63,084$ patients (mean age $47.1 \pm 18.2$ years; $50 \%$ male) who underwent emergency or elective noncardiac surgery between January $1^{\text {st }}, 2007$ and October $30^{\text {th }}, 2017$ at Vanderbilt University Medical Center. A derivation subsample reflecting two-thirds of available patients $(n=42,082)$ was randomly selected for model development, and associations were identified between predictor variables and naloxone administration occurring within 5 days following surgery. The resulting probability model for predicting naloxone administration was then cross-validated in a separate validation cohort reflecting the remaining one-third of patients $(n=21,002)$.

Results: The rate of naloxone administration was identical in the derivation $(n=2,720[6.5 \%])$ and validation $(n=1,360[6.5 \%]$ ) cohorts. The risk prediction model identified female sex (Odds Ratio [OR]: 3.01; $95 \%$ confidence interval [Cl]: 2.73-3.32), high-risk surgical procedures (OR: 4.16; 95\% Cl: 3.78-4.58), history of drug abuse (OR: $1.81 ; 95 \% \mathrm{Cl}: 1.52-2.16)$, and any opioids being administered on a scheduled rather than as-needed basis (OR: $8.31 ; 95 \% \mathrm{Cl}$ : 7.26-9.51) as risk factors for naloxone administration. Advanced age (OR: $0.40 ; 95 \% \mathrm{Cl}: 0.36-0.43$ ), opioids administered via patient-controlled analgesia pump (OR: $0.55 ; 95 \% \mathrm{Cl}: 0.49-0.62$ ), and any scheduled non-opioids (OR: $0.63 ; 95 \% \mathrm{Cl}: 0.58-0.69$ ) were associated with decreased risk of naloxone administration. An overall risk-prediction model incorporating the common clinically-available variables above displayed excellent discriminative ability both the derivation and validation cohorts (c-index $=0.820$ and 0.814 , respectively).

Conclusion: Our cross-validated clinical predictive model accurately estimates the risk of serious opioidrelated respiratory depression requiring naloxone administration in postoperative patients. In the precision medicine context, it may prove useful in facilitating selection of opioid-sparing pain management strategies for high-risk surgical patients.

\section{Introduction}

Opioid-induced respiratory depression occurs in $0.15 \%-1.1 \%$ of all surgical patients, and this risk may be increased several-fold in patients with predisposing risk factors (e.g. preoperative opioid dependence, morbid obesity, sleep apnea).[1, 2] Accurately assessing the probability of significant respiratory depression requiring naloxone administration is essential to preoperative risk assessment and planning for safe and effective postoperative pain management.

Available retrospective observational studies which have aimed to predict opioid-induced respiratory depression or oversedation requiring naloxone have several limitations.[3, 4] A two-year study investigating oversedation following opioid analgesic administration identified 53 events among patients 
above 18 years of age.[4] Being opioid naïve prior to opioid administration was the only predictor of oversedation identified, although the small sample had little statistical power for testing predictors. Generalization of this finding to the perioperative opioid administration context is limited by the fact that not all patients had undergone surgery, surgery type was not specified, and that opioid PCA administration was excluded, as well as the focus on predicting timing of oversedation relative to duration of opioid effects.

A larger $(n=163,191)$ retrospective study encompassing twelve acute care hospitals tested predictors of opioid-related respiratory depression as indexed by naloxone administration [3]. In a multivariate model, occurrence of significant respiratory depression was predicted by older age; female sex; low or high body mass index; undergoing surgery; pre-existing opioid use; and presence of chronic obstructive pulmonary disease, hepatic or renal insufficiency, or sleep apnea. In addition, the strongest predictor of naloxone administration was concurrent administration of sedating medication (most commonly, benzodiazepines). Based on these findings, the authors developed a weighted oversedation risk criteria (ORC) scoring system (range 0-24) which could be used to stratify patients as high, moderate, and low risk of requiring naloxone administration.[3] In the original derivation sample, this ORC scoring system showed good predictive value (c-index $=0.755$ ). However, neither the predictive model nor the ORC scoring system were validated in a separate sample, so it is unknown whether predictive accuracy would be similar in a new sample. Application of these findings to perioperative risk prediction are also limited by inclusion of non-operative patients in the sample, and failure to address the type of surgery, method of opioid administration, and type of opioids used.

Other retrospective studies in small samples have reported that female sex; untreated obstructive sleep apnea; long-acting oxycodone administration; history of renal-, cardiac-, or respiratory disease; concurrent use of sedatives; and smoking status all significantly predict naloxone administration in hospitalized patients who did not undergo surgery.[5, 6] One study in 225 patients identified oral and intravenous asneeded analgesia administration (compared to scheduled opioids) as factors associated with a reduced risk for opioid-induced respiratory depression.[6] Neither of these latter studies evaluated the impact of type of surgery, and neither specifically evaluated surgical patients.

The highest quality study available seeking to predict risk for opioid-induced respiratory depression (focused solely on parenteral opioids) is the recent prospective PRediction of Opioid-induced respiratory Depression In patients monitored by capnoGraphY (PRODIGY) trial [7]. In 1,335 patients, a surprisingly large proportion of patients (44\%) experienced respiratory depression as indexed by oximetry and capnography. A multivariate model indicated that age greater than 60 years, male sex, being opioid naive, presence of sleep disorder, and a history of chronic heart failure significantly predicted opioid-induced respiratory depression. This model had good predictive accuracy (c-index $=0.76$ ) and a risk score was developed. However, this risk score was not validated in a separate sample, so its predictive value outside of the derivation dataset is unknown. Moreover, while predictors of respiratory depression were identified based on objective oximetry and capnography, these outcomes do not necessarily reflect severe 
respiratory depression requiring intervention with naloxone. This trial is also limited by its sole focus on parenteral opioid administration and lack of information on impact of type of surgery.

In summary, while several studies have sought to identify factors predictive of opioid-related respiratory depression, many were carried out in relatively small samples with inadequate power, few have examined a comprehensive spectrum of comorbidities, none have specifically focused on the postsurgical patient population, impact of surgery type and method of opioid administration are not consistently addressed, and no proposed predictive model has been validated in a separate sample. The current project sought to address these limitations by developing and validating in a separate sample a simple risk prediction tool that accounts for significant patient- and procedure-related risk factors and type and administration of opioid medications for the prediction of opioid-induced respiratory depression requiring naloxone administration in patients undergoing noncardiac surgery. Early identification of patients at high risk for postoperative opioid adverse events via an automated tool incorporated in the electronic health record (EHR) could allow providers to engage in early opioid-sparing multimodal analgesia and pursue risk mitigation strategies by selecting the type and specific method of opioid associated with a decreased risk.

\section{Methods}

\section{Study Population}

The Vanderbilt University Institutional Review Board (IRB \#180634, February 17, 2020) approved this study and waived the requirement for informed consent. All methods were carried out in accordance with relevant guidelines and regulations. The pool of potential patients for this study included $n=95,396$ patients who underwent noncardiac surgery at Vanderbilt University Medical Center (Nashville, Tennessee) between January 1st, 2007, and October 30, 2017. The final sample included the subset of patients above who had perioperative data in the Vanderbilt Department of Anesthesiology Perioperative Data Warehouse, and who met the following inclusion and exclusion criteria: (1) were 18 years of older, (2) had essential clinical information on perioperative opioid and non-opioid pain medication administration available, and (3) had information available regarding whether or not they had received naloxone within 5 days after surgery. When a patient had undergone multiple surgical encounters during the designated time period, only the first encounter was included. A total of $n=84,180$ patients were included in the current study (Fig. 1).

\section{Data Collection}

We extracted data from the electronic health record (EHR) on potential clinical predictors of serious opioid-related respiratory depression events requiring naloxone administration. Based on prior work, potential predictors examined included patient demographics (age, sex), preoperative characteristics (Elixhauser comorbidity measures[8]), and perioperative opioid- and non-opioid analgesic medication administration. Use of opioid- and non-opioid analgesics was categorized by medication type, mode of 
administration, and frequency of administration based on information in the EHR. Opioid and non-opioid medications identified and included in the study are listed in Supplemental Table 1. The routes of administration were classified as intravenous bolus, intravenous continuous infusion, oral, and scheduled versus pro re nata (PRN). Finally, to ascertain the type of surgical procedure that individual patients underwent in the current study, we used Current Procedural Terminology (CPT) codes available in the EHR of the study patients [9].

\section{Serious Respiratory Depression Event Outcome}

The indicator of occurrence of a serious respiratory depression event targeted for the present study was administration of naloxone (binary outcome variable). This outcome was ascertained from the patients' EHR. To enhance specificity, this naloxone administration outcome was only considered positive when naloxone was administered within the first 5 postoperative days after a qualifying noncardiac surgery procedure.

\section{Model Development}

To derive a model predicting risk for a postoperative opioid-related respiratory depression event requiring naloxone administration, we used patient demographics, preoperative characteristics, perioperative opioid- and non-opioid pain medication administration, and surgical procedure types as defined by CPT codes (Table 1). Patients in our study population underwent a significant number of different noncardiac surgical procedures with notable differences in frequency of naloxone administration across types of procedures. Based on the frequency distribution of postoperative naloxone administration in the derivation cohort, surgical procedures were grouped into 4 risk group categories: high-risk $(4.98 \%$, naloxone administration; procedures for musculoskeletal-, respiratory-, mediastinum, diaphragm, and female genital systems), high-intermediate ( $1.23 \%$, naloxone administration; procedures for integumentary-, cardiovascular-, digestive systems), low-intermediate $(0.23 \%$, naloxone administration; procedures for male genital-, eye and ocular adnexa, nervous-, and urinary systems), and low-risk ( $0.03 \%$, naloxone administration; procedures for endocrine-, auditory-, hemic-, and lymphatic systems). This procedural risk variable was used in predictive models to permit examination of the predictive value of other preoperative predictors independent of the inherent risk of naloxone use associated with different types of surgery.

\section{Statistical Analysis}

Two-thirds $(n=42,082)$ of the total cohort was randomly assigned to the derivation cohort, which we used to develop our probability risk prediction model for serious opioid-related adverse drug events requiring naloxone administration. Using $X^{2}$ test for categorical variables and independent $t$-test for continuous variables, clinical characteristics were compared between the derivation and validation cohorts to confirm that they were similar. Multivariable logistic regression analysis with the least absolute shrinkage and selection operator (Lasso) method was used to develop a robust multivariable model while avoiding overfitting. In regression models, independent variables included patient demographics, preoperative characteristics (Elixhauser comorbidity measures[8]), perioperative opioid- and non-opioid analgesic 
medication administration, and the risk of the surgical procedure. The dichotomous dependent variable was occurrence of serious opioid-related respiratory depression requiring naloxone administration. The discriminative ability of the multivariable logistic regression model was evaluated with the concordance statistic (C-index), which is identical to the area under the receiver operating characteristics curve and ranges from 0.5 (performance by chance) to 1.0 (optimal performance).[10] The model fit of the multivariable logistic regression model was further assessed using the Hosmer-Lemeshow goodness-offit test.[11] Odds ratios and corresponding 95\% confidence intervals are reported.

Based on the findings of the analyses above, we developed a simple risk score for predicting serious opioid-related respiratory depression requiring naloxone administration. The coefficients of the predictors of the multivariable model were multiplied by 10 and rounded to the nearest integer. The weighted scores were then assigned to each categorical predictor, which were summed to allow a total risk score for each patient to be calculated. Afterwards, the total risk score was applied to a probability plot, which shows the corresponding probability of serious opioid-related adverse drug event requiring naloxone administration.

A series of subgroup analyses were also performed to determine whether the observed association between opioid medication administration and a serious opioid-related adverse drug event requiring naloxone administration was affected by a specific type or route of opioid medication administration.

All tests employed an a priori $5 \%$ type-I error rate. No familywise hypotheses were addressed. All statistical procedures were implemented in R (https://www.r-project.org) using reproducible research principles. The reporting of statistical results adhered to the guidelines provided in the STROBE (Strengthening the Reporting of Observational studies in Epidemiology)[12] and TRIPOD (Transparent Reporting of a multivariable prediction model for Individual Prognosis Or Diagnosis)[13] statements.

\section{Results}

The mean age of the entire cohort was $47 \pm 18.2$ years, and $n=31,831$ (50.5\%) of the patients were males. Serious opioid-related respiratory depression requiring naloxone administration within five days post-operatively was noted in $n=4,080(6.5 \%)$ patients. Among the $n=42,084$ patients randomly assigned to the derivation cohort, $n=2,720(6.5 \%)$ patients required and $n=39,362$ (93.5\%) patients did not require naloxone administration. Among the 21,002 patients randomly assigned to the validation cohort, $n=1,360(6.5 \%)$ patients required and $n=19,642$ (93.5\%) patients did not require naloxone administration within five days post-operatively (Fig. 1).

Figure 1. Flowchart of the study population with inclusion and exclusion criteria.

The patients who experienced serious opioid-related respiratory depression requiring naloxone administration in the derivation and validation cohorts had similar baseline and clinical characteristics except for a history of depression and any postoperative opioid administration, which were more common in the validation cohort (Table 1). 
Table 1

Patient Characteristics of the Derivation and Validation Cohorts

Derivation Cohort

Naloxone
administered

$(n=2720)$
Naloxone not administered

$(n=39362)$

\section{Validation Cohort}

Demographic

Variables

\begin{tabular}{llllll|} 
Age, years & $37 \pm 16$ & $49 \pm 18$ & $37 \pm 15$ & $49 \pm 18$ & 0.07 \\
$\begin{array}{l}\text { Sex, males } \\
\begin{array}{l}\text { Elixhauser } \\
\text { comorbidities }\end{array}\end{array}$ & $626(23.01)$ & $20536(52.17)$ & $330(24.26)$ & $10339(52.64)$ & 0.23 \\
$\begin{array}{l}\text { Congestive heart } \\
\text { failure }\end{array}$ & $58(2.13)$ & $1258(3.20)$ & $28(2.06)$ & $630(3.21)$ & 0.97 \\
$\begin{array}{l}\text { Cardiac } \\
\text { arrhythmia }\end{array}$ & $132(4.90)$ & $2788(7.08)$ & $60(4.41)$ & $1334(6.79)$ & 0.16 \\
$\begin{array}{l}\text { Pulmonary } \\
\text { circulatory } \\
\text { disorders }\end{array}$ & $33(12.13)$ & $584(1.48)$ & $10(0.74)$ & $291(1.48)$ & 0.74 \\
$\begin{array}{l}\text { Peripheral } \\
\text { vascular disease }\end{array}$ & $78(2.90)$ & $2156(5.48)$ & $48(3.53)$ & $1042(5.30)$ & 0.53 \\
$\begin{array}{l}\text { Uncomplicated } \\
\text { hypertension }\end{array}$ & $375(13.8)$ & $7679(19.51)$ & $206(15.15)$ & $3774(19.21)$ & 0.57 \\
$\begin{array}{l}\text { Complicated } \\
\text { hypertension }\end{array}$ & $16(0.59)$ & $358(0.91)$ & $4(0.29)$ & $183(0.93)$ & 0.98 \\
$\begin{array}{l}\text { Paralysis } \\
\begin{array}{l}\text { Other neurologic } \\
\text { disorders }\end{array}\end{array}$ & $22(0.81)$ & $795(2.02)$ & $14(1.03)$ & $406(2.07)$ & 0.62 \\
$\begin{array}{l}\text { Chronic } \\
\text { pulmonary } \\
\text { disease }\end{array}$ & $249(9.15)$ & $3547(9.01)$ & $107(7.87)$ & $1694(8.62)$ & 0.06 \\
$\begin{array}{l}\text { Uncomplicated } \\
\text { diabetes }\end{array}$ & $204(7.50)$ & $3546(9.01)$ & $107(7.87)$ & $1769(9.01)$ & 0.93 \\
$\begin{array}{l}\text { Complicated } \\
\text { diabetes }\end{array}$ & $66(2.43)$ & $1238(3.15)$ & $28(2.06)$ & $614(3.13)$ & 0.77 \\
$\begin{array}{l}\text { Hypothyroidism } \\
\text { Renal failure }\end{array}$ & $128(4.71)$ & $1955(4.97)$ & $65(4.78)$ & $997(5.08)$ & \\
\hline
\end{tabular}




\begin{tabular}{|c|c|c|c|c|c|}
\hline \multirow[b]{2}{*}{ Liver disease } & \multicolumn{2}{|c|}{ Derivation Cohort } & \multicolumn{3}{|c|}{ Validation Cohort } \\
\hline & $123(4.52)$ & $2122(5.39)$ & $63(4.63)$ & $1086(5.53)$ & 0.47 \\
\hline $\begin{array}{l}\text { Peptic ulcer } \\
\text { disease }\end{array}$ & $16(0.59)$ & $397(1.01)$ & $10(0.74)$ & $186(0.95)$ & 0.56 \\
\hline AIDS/HIV & $17(0.63)$ & $187(0.48)$ & $8(0.59)$ & $93(0.47)$ & 0.95 \\
\hline Lymphoma & $8(0.29)$ & $537(1.36)$ & $8(0.59)$ & $261(1.33)$ & 0.88 \\
\hline Metastatic cancer & $64(2.35)$ & $1865(4.74)$ & 27 (1.99) & $947(4.82)$ & 0.76 \\
\hline $\begin{array}{l}\text { Solid tumor } \\
\text { without } \\
\text { metastasis }\end{array}$ & $167(6.14)$ & $6286(15.97)$ & $73(5.37)$ & $3186(16.22)$ & 0.55 \\
\hline $\begin{array}{l}\text { Rheumatoid } \\
\text { arthritis }\end{array}$ & $39(1.43)$ & 760 (1.93) & $33(2.43)$ & $410(2.09)$ & 0.07 \\
\hline Coagulopathy & $126(4.63)$ & $1953(4.96)$ & $67(4.93)$ & $1013(5.16)$ & 0.27 \\
\hline Obesity & $135(4.96)$ & $1542(3.92)$ & $65(4.78)$ & 745 (3.79) & 0.43 \\
\hline Weight loss & $98(3.60)$ & $1911(4.85)$ & $61(4.49)$ & $972(4.95)$ & 0.42 \\
\hline $\begin{array}{l}\text { Fluid electrolyte } \\
\text { disorder }\end{array}$ & $366(13.46)$ & $6417(16.30)$ & $200(14.71)$ & $3150(16.04)$ & 0.59 \\
\hline Blood loss anemia & $13(0.48)$ & $181(0.46)$ & $9(0.66)$ & $97(0.49)$ & 0.45 \\
\hline Deficiency anemia & $128(4.71)$ & $1637(4.16)$ & $82(6.03)$ & 803 (4.09) & 0.91 \\
\hline Alcohol abuse & $64(2.35)$ & $1043(2.65)$ & $40(2.94)$ & $488(2.48)$ & 0.39 \\
\hline Drug abuse & $182(6.69)$ & $1291(3.28)$ & $108(7.94)$ & $676(3.44)$ & 0.14 \\
\hline Psychoses & $139(5.11)$ & $1353(3.44)$ & $67(4.93)$ & $726(3.70)$ & 0.14 \\
\hline Depression & $222(8.16)$ & $2764(7.02)$ & $131(9.63)$ & $1457(7.42)$ & 0.03 \\
\hline \multicolumn{6}{|l|}{ Surgical Procedure } \\
\hline Low risk & $12(0.44)$ & $1292(3.28)$ & $5(0.37)$ & $637(3.24)$ & 0.77 \\
\hline $\begin{array}{l}\text { Low to } \\
\text { intermediate risk }\end{array}$ & $98(3.60)$ & $5134(13.04)$ & $47(3.46)$ & $2596(13.22)$ & 0.59 \\
\hline $\begin{array}{l}\text { Intermediate to } \\
\text { high-risk }\end{array}$ & $507(18.64)$ & $18122(46.04)$ & $267(19.63)$ & 9015 (45.90) & 0.86 \\
\hline High risk & 2103 (77.32) & $14814(37.64)$ & 1041 (76.54) & 7394 (37.64) & 0.93 \\
\hline
\end{tabular}




\begin{tabular}{|llllll|}
\hline \multicolumn{4}{l}{ Derivation Cohort } & \multicolumn{2}{l|}{ Validation Cohort } \\
\hline $\begin{array}{l}\text { Any scheduled } \\
\text { opioids }\end{array}$ & $2449(90.04)$ & $23504(59.71)$ & $1209(88.90)$ & $11659(59.36)$ & 0.33 \\
\hline Any PCA opioids & $369(13.57)$ & $6200(15.75)$ & $174(12.79)$ & $3026(15.41)$ & 0.22 \\
\hline $\begin{array}{l}\text { Any scheduled } \\
\text { non-opioids }\end{array}$ & $975(35.85)$ & $14518(36.88)$ & $505(37.13)$ & $7094(36.12)$ & 0.12 \\
\hline
\end{tabular}

Values are expressed as mean ( \pm standard deviation), median [interquartile range], or $\mathrm{n}(\%) . P$ values from t-test for continuous variables, and $\chi^{2}$ of Fischer's exact test for categorical variables as appropriate.

Surgical procedures were grouped and classified according to the rate of naloxone administration as: lowrisk (endocrine-, auditory-, hemic-, and lymphatic systems), low to intermediate risk (male genital-, eye and ocular adnexa, nervous-, and urinary systems), intermediate to high-risk (integumentary-, cardiovascular-, and digestive systems), and high risk (musculoskeletal-, respiratory-, mediastinum, diaphragm, and female genital systems)

\section{Predictors of a Serious Opioid-related Adverse Drug Event Requiring Naloxone Administration}

Multivariable logistic LASSO regression indicated that younger age, female sex, high-risk surgical procedure (procedures of the musculoskeletal-, respiratory-, mediastinum, diaphragm, and female genital system), history of drug abuse, perioperative administration of scheduled (versus prn) opioids, and use of opioid-based patient-controlled analgesia and any scheduled non-opioid analgesic medication administration (the latter two associated with lower risk) were identified as significant predictors of a serious opioid-related adverse drug event requiring naloxone administration. Indeed, any scheduled (vs. prn) opioid use was the strongest predictor of postoperative naloxone administration within 5 days after surgery. This was followed by high-risk surgical procedure, female sex and history of drug abuse. In contrast, administration of any scheduled non-opioid analgesics, opioid based patient-controlled analgesia, and advanced age were factors significantly associated with a lower risk for postoperative naloxone administration within 5 days after surgery. The c-index of the model was 0.820 . However, the adjusted Hosmer-Lemeshow goodness of fit was significant for lack of fit $(P<0.05)$.

\section{Derivation of the Probability Model}

The multivariable logistic regression revealed 7 independent predictors of postoperative naloxone administration within 5 days after surgery. These variables were used to create a variable-weighted index where we assigned scores on the basis of parameter estimates of the individual predictors. By summing the individual scores from the given predictors to create a total risk score for each patient, the patient's probability of serious opioid-related adverse drug event requiring naloxone administration can be derived from Fig. 2. 


\section{Validation of the Probability Model}

All 7 individual predictors in the probability model we developed remained significantly associated with increased risk for postoperative naloxone administration within 5 days after surgery in the separate validation sample (Table 2). The overall performance of the probability model with the 7 predictors was similar in the validation sample (c-index $=0.820)$ to that observed in the derivation sample $(\mathrm{c}$-index $=$ $0.814)$.

Table 2

Multivariable predictors of a serious opioid-related adverse drug event requiring naloxone administration

\begin{tabular}{|c|c|c|c|c|}
\hline \multirow[b]{2}{*}{ Variable } & \multicolumn{2}{|c|}{$\begin{array}{l}\text { Derivation Cohort }(n= \\
\text { 42082) }\end{array}$} & \multicolumn{2}{|c|}{ Validation Cohort $(n=21002)$} \\
\hline & $\begin{array}{l}\text { Odds Ratio } \\
(95 \% \mathrm{Cl})\end{array}$ & $\begin{array}{l}P \\
\text { value }\end{array}$ & $\begin{array}{l}\text { Odds Ratio } \\
(95 \% \mathrm{Cl})\end{array}$ & $P$ value \\
\hline \multirow{3}{*}{$\begin{array}{l}\text { Female sex } \\
\text { Hiah-risk suraery }\end{array}$} & $\begin{array}{l}0.40(0.36- \\
0.43)\end{array}$ & $\dot{0} 001$ & $\begin{array}{l}0.39(0.35- \\
0.44)\end{array}$ & $<0.001$ \\
\hline & & & & $<0.001$ \\
\hline & $3.32)$ & 0.001 & $3.22)$ & $<0.001$ \\
\hline \multirow{6}{*}{$\begin{array}{l}\text { History of drug abuse } \\
\text { Any scheduled opioid } \\
\text { administration } \\
\text { Any PCA opioid administration } \\
\text { Any scheduled non-opioid } \\
\text { analgesic administration }\end{array}$} & $\begin{array}{l}4.16(3.78- \\
4.58)\end{array}$ & $<$ & $3.94(3.45-$ & $<0.001$ \\
\hline & & & & $<0.001$ \\
\hline & $\begin{array}{l}1.81(1.53- \\
2.16)\end{array}$ & $\begin{array}{l}<.001 \\
0.001\end{array}$ & $\begin{array}{l}1.82(1.44- \\
2.29)\end{array}$ & $<0.001$ \\
\hline & $\begin{array}{l}8.31(7.26- \\
9.51)\end{array}$ & $\begin{array}{l}<.001 \\
0.00\end{array}$ & $\begin{array}{l}6.88(5.73- \\
8.26)\end{array}$ & $<0.001$ \\
\hline & $\begin{array}{l}0.55(0.49- \\
0.62)\end{array}$ & $<.001$ & $\begin{array}{l}0.52(0.44- \\
0.62)\end{array}$ & \\
\hline & $\begin{array}{l}0.63(0.58- \\
0.69)\end{array}$ & $\begin{array}{l}<.001 \\
0.001\end{array}$ & $\begin{array}{l}0.72(0.63- \\
0.81)\end{array}$ & \\
\hline
\end{tabular}

$\mathrm{Cl}$, confidence interval; PCA, patient-controlled analgesia.

For definition of high-risk surgery, see the "Methods" section.

\section{Subgroup Analysis}

In the current study, our decision to characterize the frequency and mode of opioid administration as binary clinical predictors was driven by our objective to develop a simple risk index for the prediction of serious opioid-related respiratory depression requiring naloxone administration in patients undergoing noncardiac surgery. However, given the inherent bias associated with not specifying and adjusting for the type of opioid and mode of opioid administration, we also performed a series of subgroup analyses addressing these variables (Table 3 ). 
Table 3

Association between type- and route of opioid administration and the risk of a serious opioid-related adverse drug event requiring naloxone administration in the discovery cohort, and the discriminatory ability of the models in the discovery and validation cohorts.

Discovery cohort

Odds ratio
$(95 \% \mathrm{Cl})$

\section{Validation cohort}

\section{$\begin{array}{ll}P & \text { C- } \\ \text { value } & \text { index }\end{array}$}

Odds ratio

$(95 \% \mathrm{Cl})$
$\begin{array}{ll}P & \text { C- } \\ \text { value } & \text { index }\end{array}$

Model with type and any route of opioid administration adjusted for age, sex, high-risk procedure, and any scheduled non-opioid medication administration

$\begin{array}{lllllll}\text { Combined opioid } & 1.48(1.35- & < & 0.817 & 1.37(1.20- & < & 0.812 \\ \text { administration } & 1.62) & 0.0001 & & 1.56) & 0.0001 & \end{array}$

Fentanyl

$\begin{array}{lllll}\text { Oxycodone } & 1.30(1.18- & < & 1.12(0.97- & 0.1042 \\ & 1.43) & 0.0001 & 1.28) & \\ \text { Morphine } & 4.57(4.15- & < & 4.61(4.03- & < \\ & 5.02) & 0.0001 & 5.27) & 0.0001\end{array}$

Model with routes of fentanyl administration adjusted for age, sex, high-risk procedure, and any scheduled non-opioid medication administration

$\begin{array}{llllll}\text { Fentanyl administration } & 1.96(1.64- & < & 0.796 & 1.96(1.52- & < \\ \text { Scheduled } & 2.37) & 0.0001 & 2.52) & 0.0001 & 0.793 \\ \text { As needed } & 1.48(1.23- & < & 1.38(1.07- & 0.0138 \\ \text { Patient-controlled } & 1.80) & 0.0001 & 1.79) & \\ \text { analgesia } & 0.51(0.44- & < & 0.54(0.45- & 0.0001\end{array}$

Model with routes of oxycodone administration adjusted for age, sex, high-risk procedure, and any scheduled non-opioid medication administration

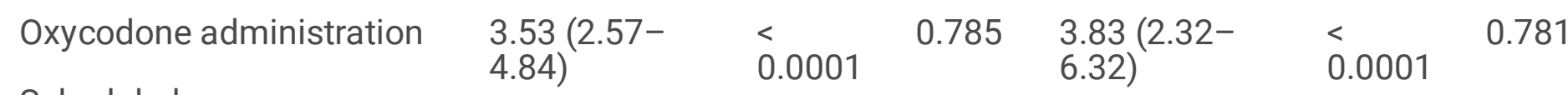

Scheduled

As needed
$0.57(0.42-\quad 0.0005$
$0.44(0.27-$
0.0013

Patient-controlled

$0.78)$

analgesia

$0.51(0.43-$

$<$

0.0001

$0.73)$

$0.53(0.41-\quad<0.0001$ 


\section{Discovery cohort}

$\begin{array}{llllll}\text { Morphine administration } & 4.25(3.06- & < & 0.808 & 5.63(3.66- & < \\ \text { Scheduled } & 5.91) & 0.0001 & 8.65) & 0.0001 & 0.802 \\ \text { As needed } & 1.71(1.23- & 0.0013 & 1.22(0.80- & 0.3603 \\ \text { Patient-controlled } & 2.37) & & 1.88) & \\ \text { analgesia } & 0.31(0.27- & 0.0001 & 0.33(0.27- & 0.0001\end{array}$

$\mathrm{Cl}$, confidence interval

\section{Validation cohort}

$5.63(3.66-$

$1.22(0.80-$ $1.88)$

$0.41)$

Our results indicated that any fentanyl-, oxycodone-, and morphine administration were all significantly associated with a higher risk for a serious opioid-related adverse drug event requiring naloxone administration (Table 3). When the type and route of opioids administered were separately studied, we found that for most of the studied opioids, PCA administration was associated with a lower risk and scheduled or as needed administration of opioids via any route were associated with a higher risk for serious opioid-related respiratory depression requiring naloxone administration (Table 3).

\section{Discussion}

We have developed and validated a simple prediction tool that can be used to estimate the risk of a serious opioid-related adverse drug event requiring naloxone administration in patients undergoing noncardiac surgery. We have demonstrated that several previously identified clinical determinants of postoperative acute pain severity (e.g., younger age, female gender, history of drug abuse, type of surgery) were also predictors of a serious opioid-related adverse drug event requiring naloxone administration in our sample of patients undergoing noncardiac surgery [14]. Based on the type and frequency of perioperative opioid and non-opioid pain medication administration obtained from the EHR, we were able to further refine our prediction model to increase its predictive ability for a serious opioid-related adverse drug event requiring naloxone administration. Finally, our subgroup analyses revealed that the type and route of opioid medication administration significantly impacted the risk of a serious opioid-related adverse drug event requiring naloxone administration.

The present study showed that a history of drug abuse, any scheduled opioid administration, younger age, and female sex were associated with increased risk for a serious opioid-related adverse drug event requiring naloxone administration after noncardiac surgery. In contrast, use of opioid-based patientcontrolled analgesia and any scheduled non-opioid analgesic medication administration were linked to lower risk of serious respiratory depression. The identified set of predictors, which replicated in an independent sample, have both similarities and differences to predictors identified in prior studies.

The adverse impact of scheduled opioids on risk of severe respiratory depression noted in the present study is similar to prior work [6]. Several existing studies have also reported that female sex predicts respiratory depression as in the current work $[3,5,6,15]$. In contrast, three studies, including the only 
available prospective study, suggested that males were at elevated risk of respiratory depression. Differences regarding sex effects on respiratory depression between some prior studies and the current work may be due in part to the specific outcome measures targeted, which included respiratory depression indexed by oximetry and capnography [7] and opioid adverse events more broadly defined [16], rather than severe respiratory depression requiring naloxone as in the current work. Another difference between the present results and prior work relates to the impact of age, with the current study indicating higher respiratory depression risk in younger individuals but some other studies showing increased risk in older individuals $[3,7,15]$. As above, differences in outcome definitions may have contributed to these different predictive patterns $[7,15]$. While reasons for these differences cannot be conclusively determined, the fact that the current predictive findings were replicated in a separate validation sample lend them credence. Finally, our finding that history of drug abuse predicted risk for a serious opioid-related adverse drug event requiring naloxone administration is similar to results of a recent large-scale retrospective study.[16] This finding may relate to the fact that for patients with a history of daily drug abuse, a higher dose of opioids is needed after surgery, thereby increasing the risk of adverse events [15].

Our findings that females are at increased risk of serious respiratory depression following surgery may be viewed within the larger context of sex differences in pain responsiveness. Studies of experimentallyinduced pain have observed that women exhibited greater pain sensitivity, enhanced pain facilitation (i.e., central sensitization), and reduced pain inhibition compared with men [17]. There is also some evidence suggesting sex differences in responses to pharmacological and non-pharmacological pain management strategies [18, 19]. Furthermore, gender biases in pain assessment and treatment appear to exist [20], which could significantly impact perioperative pain management practices and related outcomes of patients undergoing surgery [17]. Findings of sex differences in risk for respiratory depression may provide support for possible sex-specific tailoring of perioperative pain management approaches of patients undergoing noncardiac surgery.

The present findings that more advanced age, postoperative PCA, and any scheduled non-opioid analgesic administration appeared to significantly reduce the risk for serious opioid-related respiratory depression requiring naloxone administration should be considered within the context of clinical practice. Several guidelines and clinical studies recommended that the routine use of opioid analgesics should be minimized in elderly due to increased opioid sensitivity and a higher risk for cardiorespiratory complications. Thus, the observation in our study that advanced age was associated with a lower risk for serious respiratory depression may be explained by this ongoing change in routine perioperative pain management practices that incorporate greater focus on non-opioid pain management strategies in elderly surgical patient populations [21, 22].

Patients requiring significant around-the-clock postoperative intravenous opioid administration more frequently than every three to four hours could significantly benefit from PCA-based opioid administration. If used properly, opioid administration through a PCA pump may reduce the risks associated with perioperative opioid administration since patients are less likely over- or undertreated 
with opioids [23]. Indeed, our study demonstrated that continuous intravenous infusion of opioids with a PCA option was associated with a reduced risk for a serious opioid-related respiratory depression requiring naloxone administration. Nevertheless, the use of continuous intravenous infusion of opioids with PCA could potentially expose some patients to an elevated risk for other serious opioid-related adverse events [24]. Therefore, safe PCA prescribing should be standardized and include measures such as information on loading dose, consideration of a basal rate, lockout intervals, titration, and transitioning off PCA. In addition, safe PCA administration should include ongoing clinical monitoring to further minimize the risk for serious opioid-related adverse drug events [25].

In our study, we observed that any scheduled non-opioid analgesic administration was associated with a reduced risk for serious respiratory depression requiring naloxone administration. Non-steroidal antiinflammatory drugs reduce pain and inflammation by mechanisms different from that of opioid analgesics, and thus, are potentially useful in reducing the need for opioid administration as part of a multimodal analgesia strategy. Indeed, a recent meta-analysis demonstrated that administration and type of NSAIDs use were associated with $9 \%-50 \%$ reduction in opioid use [26]. Nonetheless, studies included in this meta-analysis found no difference in the frequency of severe opioid-related adverse drug events (broadly defined) in patients administered NSAIDs compared with controls. Additionally, several of these studies noted lower rates of mild adverse drug events such as nausea, vomiting, sedation and pruritus with NSAIDs administration compared to placebo. These prior findings as well as the current work indicate that NSAIDs may have an important role in reducing postoperative opioid requirements, and thus, the risk for a serious opioid-related adverse drug events.

The clinical applicability of our predictive algorithm in the perioperative management of patients undergoing noncardiac surgery should be considered along with the current recommendations of the National Action Plan for Adverse Drug Prevention for reducing the risk for opioid-related adverse drug events.[27] System-wide changes are considered the most important target for opioid-related adverse drug event prevention, and many opioid-related adverse drug events occur from overprescribing, medication errors, and inadequate monitoring of patient responses. Therefore, clinical application of our validated predictive algorithm could not only facilitate identification of patients at high risk for opioidrelated respiratory depression requiring naloxone administration, but could also contribute to system-wide practice changes resulting in lower doses of opioids prescribed and greater use of non-opioid analgesics. This could contribute to improved patient satisfaction and also reduce the risk for persistent opioid use after surgery.

Our study has some limitations. First, our study was an observational study that relied on administrative data and medical records based on physician documentation and billing codes of significant comorbidities. Hence, the effects of some of the risk factors as potential determinants of serious opioidrelated respiratory depression requiring naloxone administration may be biased. However, the predictive values of these clinical risk factors were like those identified and described in the contemporary literature $[7,28]$. Second, our probability model was based on a noncardiac surgery population at a quaternary medical center. Thus, the rates of serious opioid-related adverse drug events requiring naloxone 
administration may appear higher than those reported from other studies $(0.5-1.4 \%)[16,29]$. However, these studies with lower naloxone administration rates usually were either smaller scale studies, lacked information on timing of a serious opioid-related adverse drug event relative to opioid administration (limited to 5 days postoperatively in the current work), included a shorter postoperative observation period for naloxone administration, or selected surgical patients at low risk for serious opioid-related adverse drug events requiring naloxone administration. Finally, we were not able to study any other documented opioid-related adverse drug events that did not require naloxone administration. Typically, in a retrospective study such as ours, less severe opioid-related adverse drug events not requiring naloxone administration are ascertained based on ICD codes of these adverse events. Using ICD codes may erroneously over capture or not capture these less severe opioid-related adverse events. Thus, in our study we were not able to develop and validate our predictive model for predicting the risk of mild and moderate adverse opioid-related adverse drug events not requiring naloxone administration.

\section{Conclusions}

In conclusion, our study showed that a combination of patient characteristics, clinical risk factors, type of opioid and non-opioid medication administration are significant determinants of serious opioid-related respiratory depression requiring naloxone administration. The derived and validated clinical algorithm in our probability model is a simple risk assessment tool with good discriminative ability that we plan to incorporate into our institutional EHR as a clinical decision support tool. This is intended to help clinicians prospectively estimate and refine the probability of serious postoperative opioid-related respiratory depression after noncardiac surgery.

\section{Abreviations}

$\mathrm{Cl}$, Confidence Interval

EHR, electronic health record

ICD, International Classification of Diseases

OR, Odds Ratio

ORC, Oversedation Risk Criteria

PCA, Patient Controlled Analgesia

PRN, "Pro Re Nata" or "As Needed"

\section{Declarations}

\section{Ethics approval and consent to participate}


This study was approved by the Vanderbilt University Institutional Review Board (IRB \#180634, February $17,2020)$ approved this study and waived the requirement for informed consent. All methods were performed in accordance with the relevant guidelines and regulations.

\section{Consent for publication}

Not applicable.

\section{Availability of data and materials}

The data that support the findings of this study are available from corresponding author, but restrictions apply to the availability of these data, which were used under license for the current study, and so are not publicly available. Data are however available from the authors upon reasonable request and with permission of corresponding author.

\section{Competing interests}

The authors declare that they have no competing interests.

\section{Funding}

This work was supported by grant \#R01DA050334 (Dr. Bruehl) from the National Institute on Drug Abuse/National Institutes of Health, and the Department of Defense, Congressionally Directed Medical Program CDMRP W81XWH-15-2-0046 (Dr. van De Ven, Shaw and Kertai).

\section{Authors' contribution}

RS, XF, MSS, MDK: data collection and analyses; SB, TVDV, ADS, MDK: Conceptualization; SR, SB, TVDV, ADS, MDK: Drafting manuscript, review \& editing. All authors read and approved the final manuscript.

\section{Acknowledgments}

Part of this work was presented at the American Society of Anesthesiologists Annual Meeting in October, 2021, San Diego, California.

\section{Authors' information}

${ }^{1}$ Departments of ${ }^{1}$ Anesthesiology, Vanderbilt University Medical Center, Nashville, TN, USA; ${ }^{2}$ Department of Biostatistics, Vanderbilt University Medical Center, Nashville, TN, USA; ${ }^{3}$ Division of Pain Medicine, Duke University Department of Anesthesiology, Durham, NC, USA; ${ }^{4}$ Department of Intensive Care and Resuscitation, Cleveland Clinic, Cleveland, $\mathrm{OH}$, USA

\section{References}


1. Dahan, A., L. Aarts, and T.W. Smith, Incidence, Reversal, and Prevention of Opioid-induced Respiratory Depression. Anesthesiology, 2010. 112(1): p. 226-38.

2. Menendez, M.E., D. Ring, and B.T. Bateman, Preoperative Opioid Misuse is Associated With Increased Morbidity and Mortality After Elective Orthopaedic Surgery. Clin Orthop Relat Res, 2015. 473(7): p. 2402-12.

3. Garrett, J., et al., Predicting opioid-induced oversedation in hospitalised patients: a multicentre observational study. BMJ Open, 2021. 11(11): p. e051663.

4. Garrett, J.S., et al., Timing of Oversedation Events Following Opiate Administration in Hospitalized Patients. J Clin Med Res, 2021. 13(5): p. 304-308.

5. Pawasauskas, J., et al., Predictors of naloxone use for respiratory depression and oversedation in hospitalized adults. Am J Health Syst Pharm, 2014. 71(9): p. 746-50.

6. Brant, J.M., et al., Predictors of oversedation in hospitalized patients. Am J Health Syst Pharm, 2018. 75(18): p. 1378-1385.

7. Khanna, A.K., et al., Prediction of Opioid-Induced Respiratory Depression on Inpatient Wards Using Continuous Capnography and Oximetry: An International Prospective, Observational Trial. Anesth Analg, 2020. 131(4): p. 1012-1024.

8. Elixhauser, A., et al., Comorbidity measures for use with administrative data. Med Care, 1998. 36(1): p. 8-27.

9. Pendergrass, S.A. and D.C. Crawford, Using Electronic Health Records To Generate Phenotypes For Research. Curr Protoc Hum Genet, 2019. 100(1): p. e80.

10. Hanley, J.A. and B.J. McNeil, The meaning and use of the area under a receiver operating characteristic (ROC) curve. Radiology, 1982. 143(1): p. 29-36.

11. Lemeshow, S. and D.W. Hosmer, Jr., A review of goodness of fit statistics for use in the development of logistic regression models. American journal of epidemiology, 1982. 115(1): p. 92-106.

12. von Elm, E., et al., The Strengthening the Reporting of Observational Studies in Epidemiology (STROBE) statement: guidelines for reporting observational studies. J Clin Epidemiol, 2008. 61(4): p. 344-9.

13. Collins, G.S., et al., Transparent Reporting of a multivariable prediction model for Individual Prognosis or Diagnosis (TRIPOD): the TRIPOD statement. Ann Intern Med, 2015. 162(1): p. 55-63.

14. Kalkman, J.C., et al., Preoperative prediction of severe postoperative pain. Pain, 2003. 105(3): p. 415423.

15. Cauley, C.E., et al., Predictors of In-hospital Postoperative Opioid Overdose After Major Elective Operations: A Nationally Representative Cohort Study. Ann Surg, 2017. 265(4): p. 702-708.

16. Shafi, S., et al., Association of Opioid-Related Adverse Drug Events With Clinical and Cost Outcomes Among Surgical Patients in a Large Integrated Health Care Delivery System. JAMA Surg, 2018. 153(8): p. 757-763. 
17. Bartley, E.J. and R.B. Fillingim, Sex differences in pain: a brief review of clinical and experimental findings. Br J Anaesth, 2013. 111(1): p. 52-8.

18. Pisanu, C., et al., Sex differences in the response to opioids for pain relief: A systematic review and meta-analysis. Pharmacol Res, 2019. 148: p. 104447.

19. Gazerani, P., A.M. Aloisi, and H. Ueda, Editorial: Differences in Pain Biology, Perception, and Coping Strategies: Towards Sex and Gender Specific Treatments. Front Neurosci, 2021. 15: p. 697285.

20. Samulowitz, A., et al., "Brave Men" and "Emotional Women": A Theory-Guided Literature Review on Gender Bias in Health Care and Gendered Norms towards Patients with Chronic Pain. Pain Res Manag, 2018. 2018: p. 6358624.

21. Oderda, G.M., et al., Opioid-related respiratory and gastrointestinal adverse events in patients with acute postoperative pain: prevalence, predictors, and burden. J Pain Palliat Care Pharmacother, 2019. 33(3-4): p. 82-97.

22. Chou, R., et al., Management of Postoperative Pain: A Clinical Practice Guideline From the American Pain Society, the American Society of Regional Anesthesia and Pain Medicine, and the American Society of Anesthesiologists' Committee on Regional Anesthesia, Executive Committee, and Administrative Council. J Pain, 2016. 17(2): p. 131-57.

23. McNicol, E.D., M.C. Ferguson, and J. Hudcova, Patient controlled opioid analgesia versus non-patient controlled opioid analgesia for postoperative pain. Cochrane Database Syst Rev, 2015(6): p. CD003348.

24. Schein, J.R., et al., Patient-controlled analgesia-related medication errors in the postoperative period: causes and prevention. Drug Saf, 2009. 32(7): p. 549-59.

25. Frederickson, T.W., et al. Reducing adverse drug events related to opioids implementation guide. 2015. 1-96.

26. Martinez, L., E. Ekman, and N. Nakhla, Perioperative Opioid-sparing Strategies: Utility of Conventional NSAIDs in Adults. Clin Ther, 2019. 41(12): p. 2612-2628.

27. Ducoffe, A.R., et al., National Action Plan for Adverse Drug Event Prevention: Recommendations for Safer Outpatient Opioid Use. Pain Med, 2016. 17(12): p. 2291-2304.

28. Minhaj, F.S., et al., Predictors of Serious Opioid-Related Adverse Drug Events in Hospitalized Patients. J Patient Saf, 2020.

29. Gupta, K., et al., Risk factors for opioid-induced respiratory depression in surgical patients: a systematic review and meta-analyses. BMJ Open, 2018. 8(12): p. e024086.

\section{Figures}




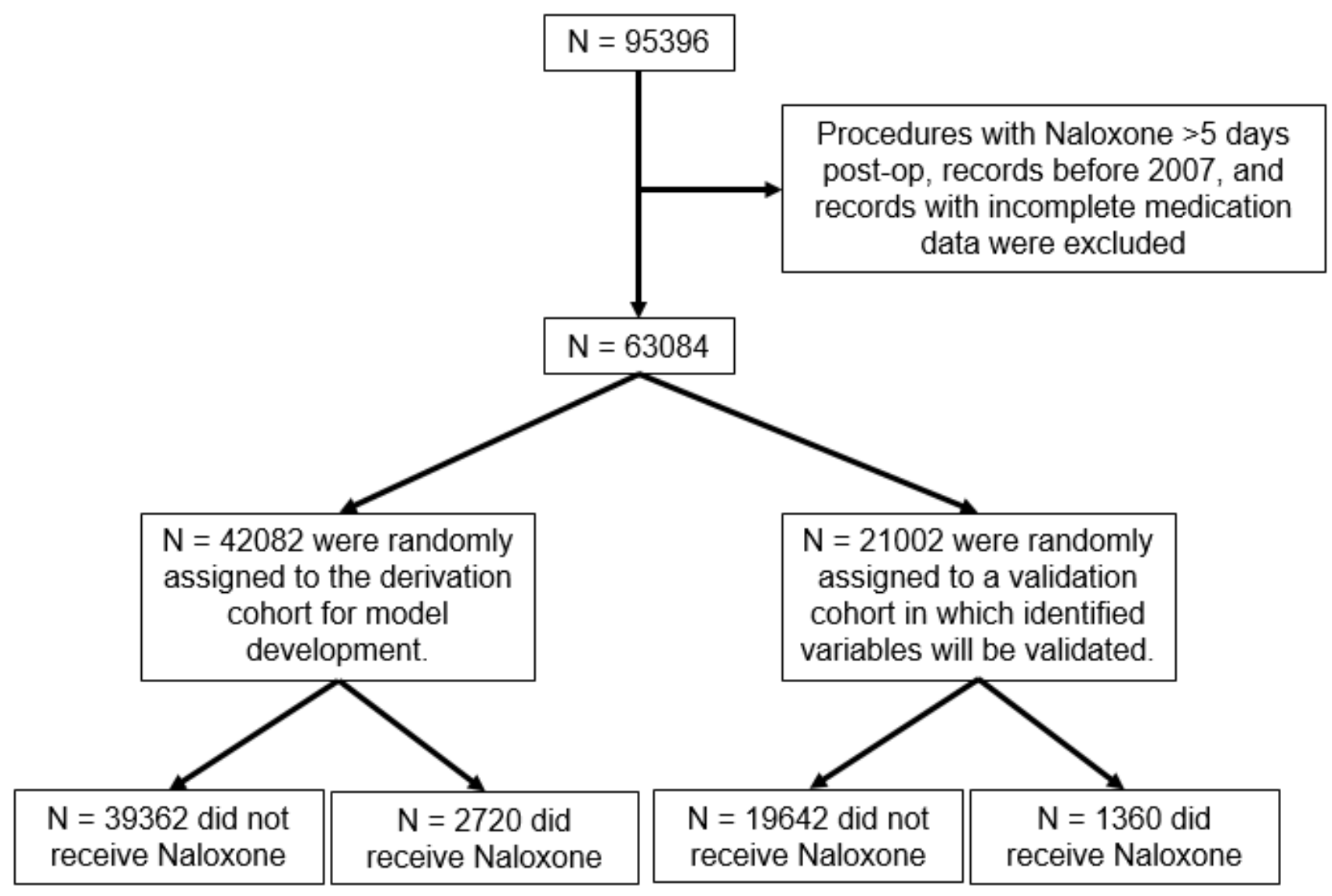

Figure 1

Flowchart of the study population with inclusion and exclusion criteria 
Estimated Probability of a Serious Opioid-Related Adverse Drug Event Requiring Naloxone Administration

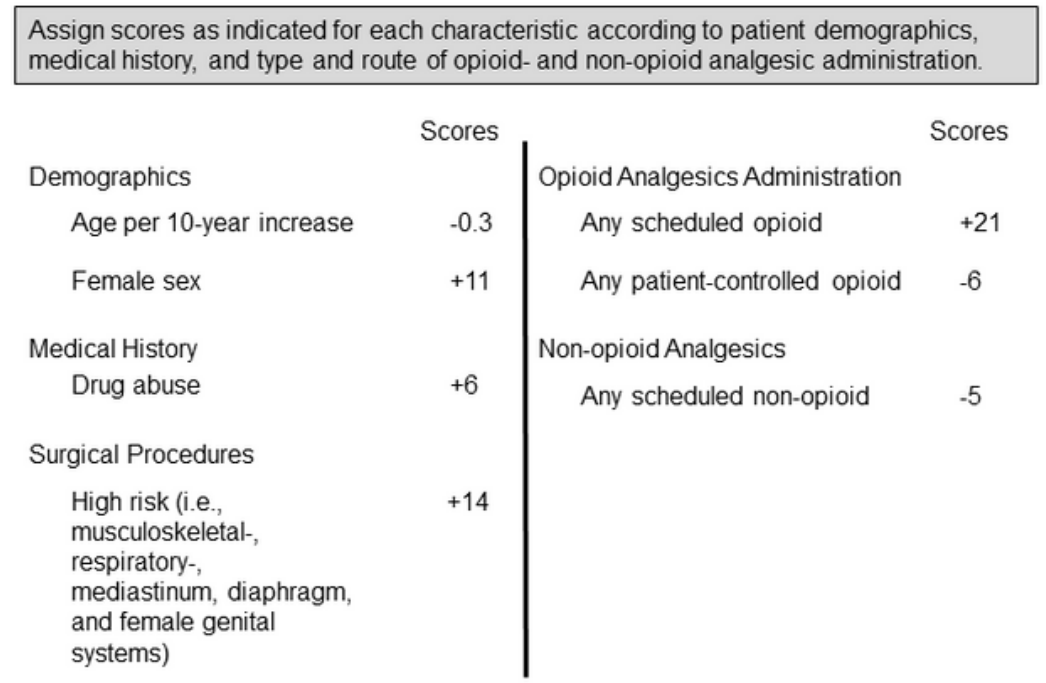

Calculate the total score by summing the individual scores from the given characteristics and, using the total risk score, read the corresponding estimated probability of a serious opioid-related adverse drug-event requiring naloxone administration.

Characteristics

Scores

Demographics

Medical History

Surgical Procedures

Opioid Analgesics Administration

Non-opioid Analgesics

Total Risk Score

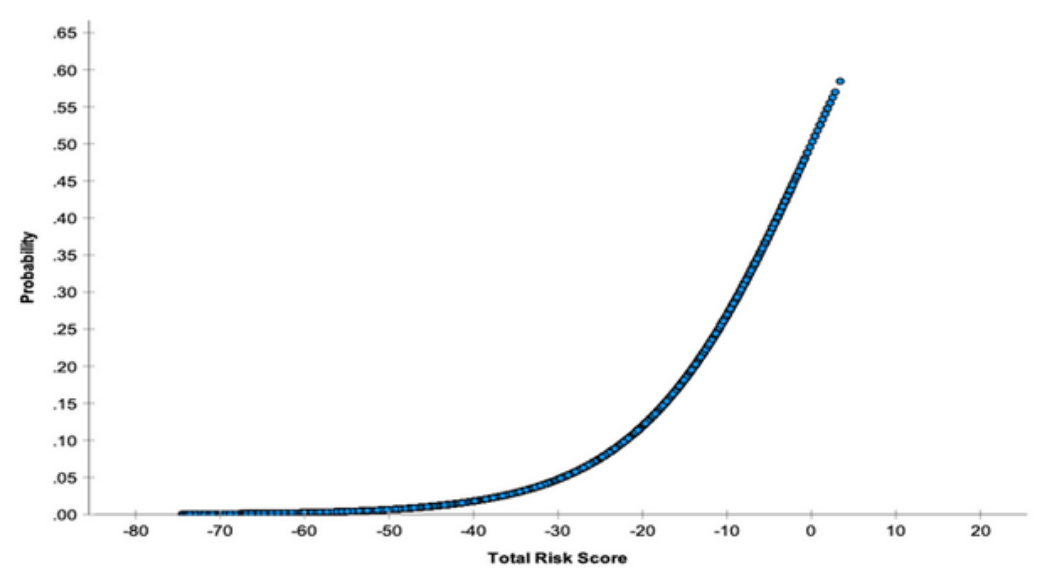

Figure 2

Probability model for a serious opioid-related adverse drug event requiring naloxone administration

\section{Supplementary Files}

This is a list of supplementary files associated with this preprint. Click to download. 
- SupplementalTable115Feb2022.docx 